\title{
A Rare Case of Adrenal Arterio-Venous Malformation (AVM): A Case Report
}

\author{
Rosanna Mamone $^{1}$ | Rocco Minelli2* ${ }^{2 *}$ Maria G. Giagnorio $^{2}$ | Sonia Tamasi ${ }^{1}$ | Eugenio Rossi ${ }^{3}$ | Donatella \\ Irace $^{4}$ | Domenico Noviello ${ }^{1}$ | Francesco Esposito ${ }^{1}$ | Anna M Giugliano ${ }^{1}$ | Massimo Zeccolini $^{1}$ | Gianfranco \\ Vallone $^{2}$
}

*Correspondence: Rocco Minelli

Address: ${ }^{1}$ U.O.C. of General Radiology P.O. Santobono-A.O.R.N. Santobono-Pausilipon, Via M. Fiore 6, 80128, Naples, Italy; 2Department Life and Health “V. Tiberio", University of Molise, Via Francesco De Sanctis 1, Campobasso 86100, Italy; ${ }^{3}$ U.O.S.D. Radiology and Ultrasound P.O. Pausilipon-AORN Santobono-Pausilipon, Via Posillipo 226, 80123 Naples, Italy; ${ }^{4}$ A.O.U. Federico II, Department of Translational Medical Sciences, Pediatric Section, Via S. Pansini, 5, Naples, Italy e-mail $\bowtie$ : rocco.minelli@libero.it

Received: 01 June 2020; Accepted: 09 June 2020

Copyright: (C) 2020 Mamone R. This is an open-access article distributed under the terms of the Creative Commons Attribution License, which permits unrestricted use, distribution, and reproduction in any medium, provided that the original work is properly cited.

\section{ABSTRACT}

Arterio-venous malformations (AVMs) are congenital vascular lesions in which dysplastic arteries are connected to the veins without an intervening capillary bed. High arterial flow directly into venous structures can lead to disruption of the venous walls and ultimately cause hemorrhage. These malformations can be found in the muscles, subcutaneous fat, bone and mainly in the central nervous system. We describe a rare case of adrenal AVM in a 6-year-old boy who arrives at the emergency room of the "Santobono" hospital.

Keywords: AVM, Adrenal Gland, Ultrasound, Pediatric

\section{Introduction}

AVMs are congenital high-flow vascular malformations characterized by direct communication between dysplastic arteries and veins in the absence of an intervening capillary bed. This means that blood passes from the arterial to the venous district with high flow and high pressure, being able to cause the rupture of the venous walls with consequent bleeding (Ozpinar et al., 2017). While these malformations were traditionally thought to represent congenital lesions resulting from disordered embryogenesis, other studies have supported the notion that they can also develop postnatally (Chen $e t$ al., 2014). AVMs are mainly found in the muscles, subcutaneous fat, bone and cranium (Kollipara et al., 2013); these lesions are most commonly found in central nervous system and less frequently on limbs and trunk (Stapf et al., 2001). Ultrasound and, in particular color-Doppler sonography, allow to diagnose AVMs through the identification of vessels with high systolic and diastolic flow (Dubois and Garel, 1999), while CT can provide useful informations on the involvement of adjacent organs and on the extent of the mass (Samadi and Salazar, 2019). 


\section{Case Report}

6-year-old boy arrives at the "Santobono" Pediatric Hospital for abdominal pain. In the anamnesis there is a prenatal diagnosis of neoformation in the left hemithorax, framed as a probable lung seizure and not further investigated after birth. On abdominal physical examination, the abdomen is treatable and painless on palpation. An ultrasound of the abdomen is performed in the first instance which shows in the subdiaphragmatic area, above the left kidney and dissociable from it, a non-homogeneous, roughly triangular area of about $4 \mathrm{~cm}$ of max transverse diameter (Fig. 1).

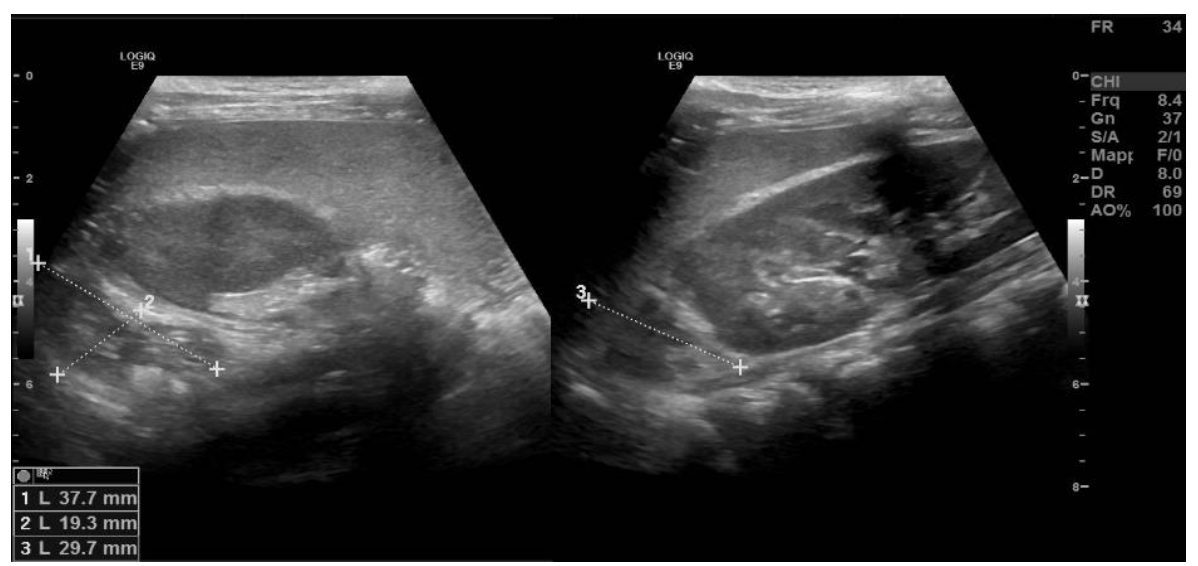

Figure 1: Longitudinal scan that shows in the subdiaphragmatic area, above the left kidney and dissociable from it, a non-homogeneous, roughly triangular area of about $4 \mathrm{~cm}$ of transverse diameter max

Given the unequivocal interpretation of this finding and considering the presumed prenatal diagnosis of lung seizure, a thoraco-abdominal CT is performed with and without contrast medium that confirms a hypodense area with left adrenal seat at the pre-contrast scans (Fig. 2a) and with uneven enhancement in the post-contrast phase (Fig. 2b).

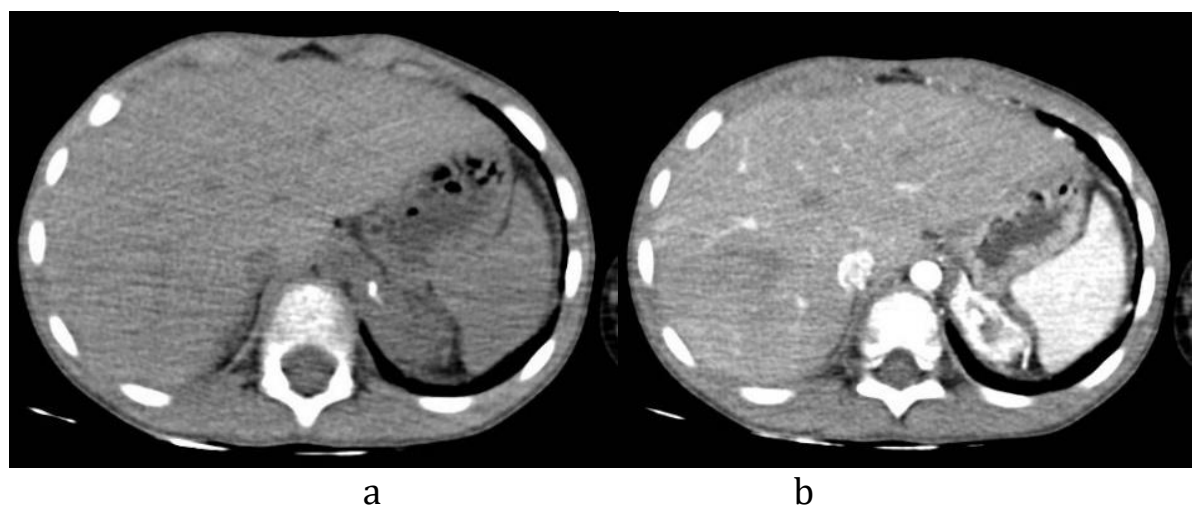

Figure 2(a-b): a) Axial CT scan without contrast showing hypodense area with left adrenal site b) Axial CT scan with contrast showing uneven enhancement in the post-contrast phase

This neoformation appears to be connected by two fine arterial vascular structures, one of aortic origin (Fig. 3). 


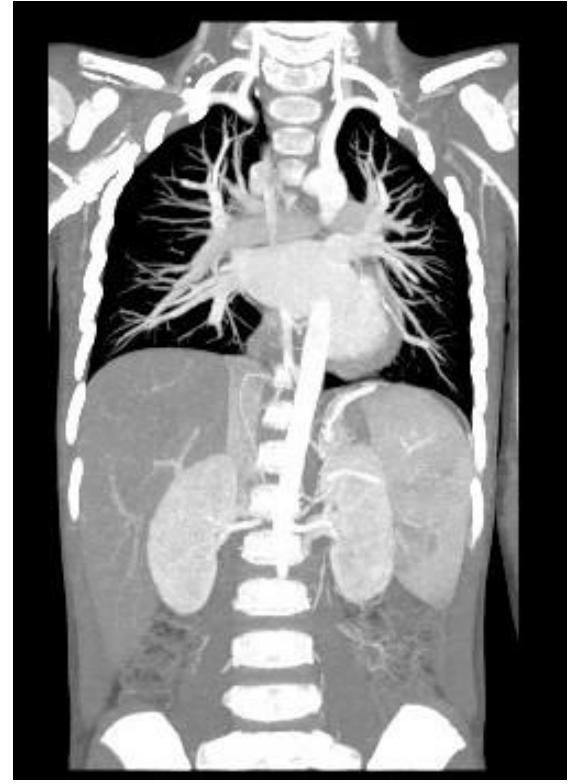

Figure 3: Coronal CT scan with MIP reconstruction that highlights a neoformation connected by two fine vascular structures of an arterial nature, one of aortic origin, the other to the celiac tripod

The other to the celiac tripod, while another larger vessel of a venous nature drains into the subcostal vessels (Fig. 4).

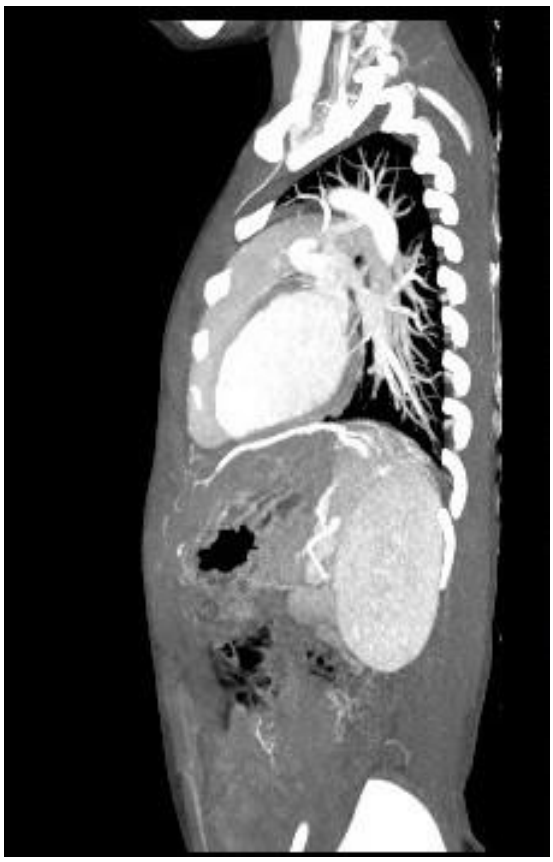

Figure 4: Sagittal CT scan with MIP reconstruction showing another vessel of a larger caliber of venous nature that drains into the subcostal vessels

The finding appears to be compatible with the diagnosis of arteriovenous malformation. The abdomen ultrasound is then repeated with a targeted evaluation of the vascularization in the splenicadrenal lodge. The color-Doppler control demonstrates numerous vascular poles distributed over the whole lesion (Fig. 5). 


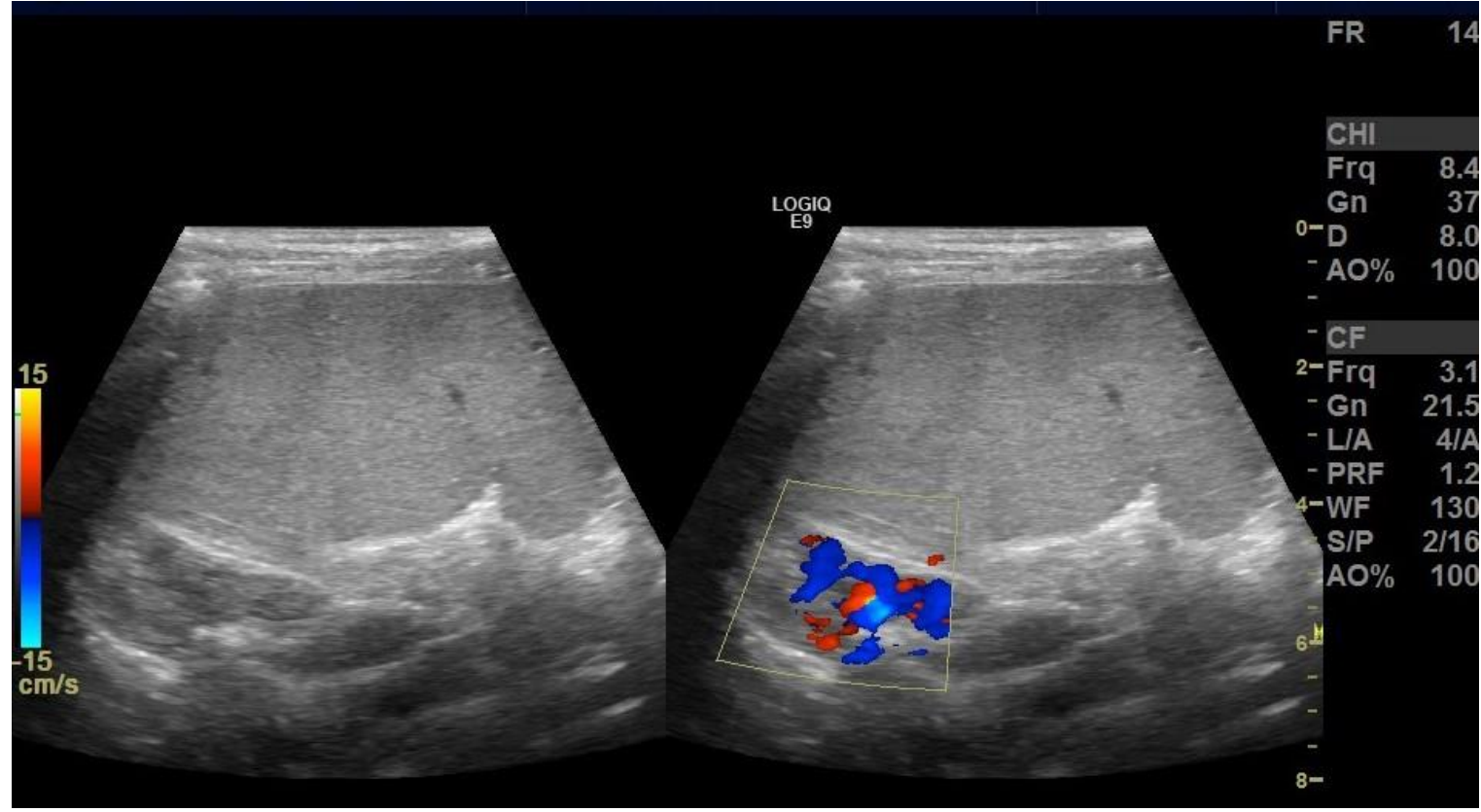

Figure 5: Longitudinal color-Doppler ultrasound scan demonstrating numerous vascular poles distributed throughout the lesion

and the velocimetric analysis shows a prevalence of venous vessels with arterialized flow (Fig. 6).

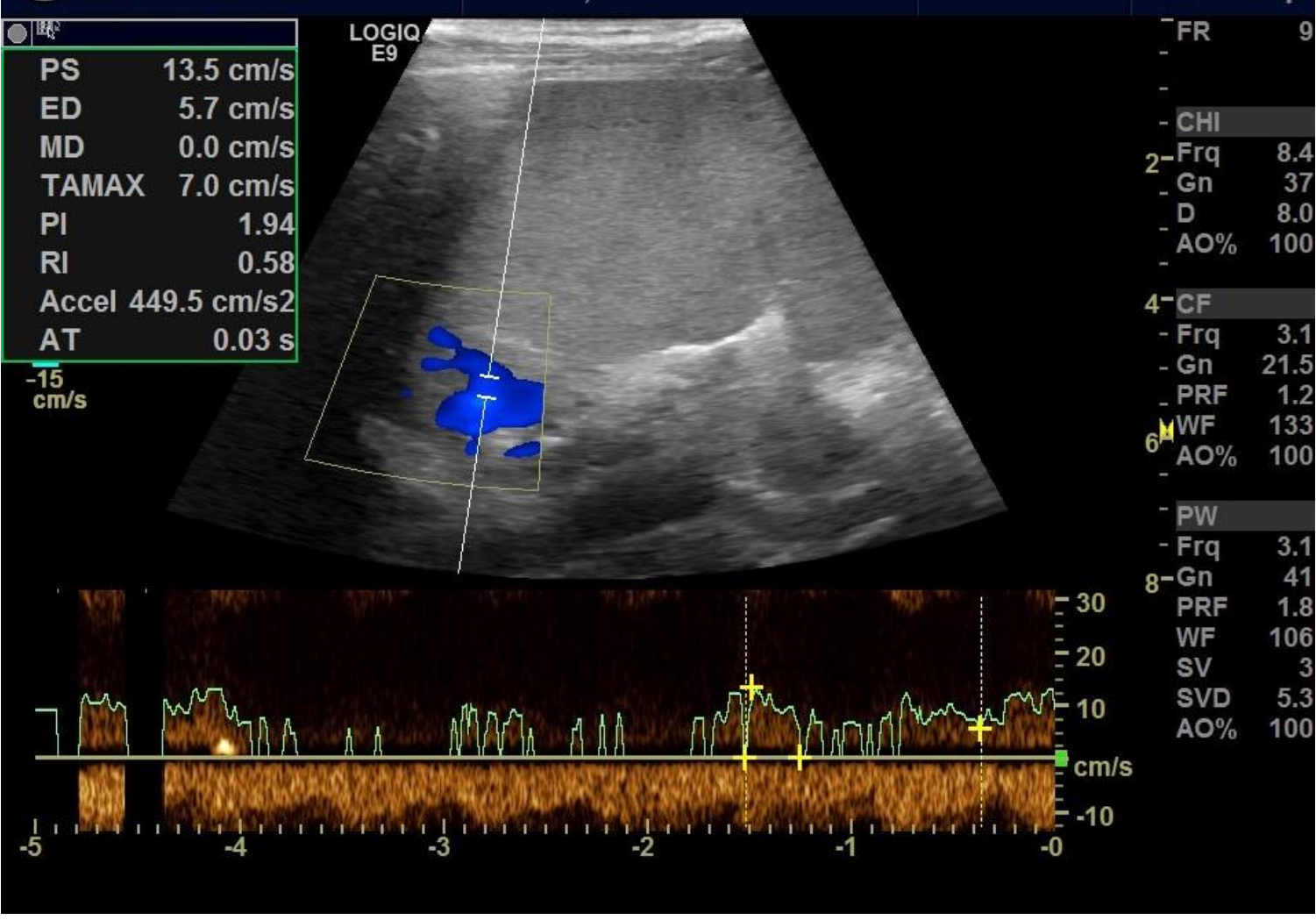

Figure 6: Longitudinal ultrasound scan that the velocimetric analysis shows a prevalence of venous vessels with arterialized flow

The diagnosis of adrenal arteriovenous malformation is therefore confirmed. 


\section{Discussion}

AVMs are vascular malformations characterized by direct and pathological communication between arteries and veins. They are high-flow vascular malformations because blood passes from the arterial to the venous district with high pressure, along abnormal, dilated and tortuous vascular channels that form a sort of ball called nidus. Therefore AVM is defined by one or more afferent arteries, a nidus and drainage veins (Conway and Rosen, 2018). Children make up only a fifth of AVM patients, although these malformations account for $20-50 \%$ of spontaneous intracranial haemorrhages when they are in the central nervous system (Brillantino et al., 2019). The aetiopathogenesis is still not completely understood, but the lesions would appear to be present from birth and to derive from anomalies in the expression of certain growth factors during angiogenesis (Rangel-Castilla et al., 2014). However, there are studies that support the idea that AVMs can be lesions acquired after birth as consequence of traumatic vascular damage or venous thrombotic phenomena (Chen et al., 2014). These malformations are mainly found in the muscles, subcutaneous fat, bone and cranium (Kollipara et al., 2013); these lesions are most commonly found in central nervous system and less frequently on limbs and trunk (Stapf et al., 2001). The clinical picture therefore varies according to the location of the AVM. When they are localized in soft tissues, they appear as a swelling of variable proportions and extension, with a soft-elastic consistency, with palpatory appreciable thrill and with a certain degree of locoregional hypertrophy. In case of cerebral localization, they can remain asymptomatic or cause headache, epileptic seizures, neurological deficits and cerebral hemorrhages if the high arterial flow causes breakdown of venous walls (Tufano et al., 2020). Different is the case in which AVMs have an atypical site, as in our case, so they can remain asymptomatic for long periods and the diagnosis can be incidental during diagnostic investigations performed for other reasons. Imaging has an important role in the diagnosis of AVM. In particular, ultrasound is the first choice examination in the study of vascular anomalies for its availability, low cost and the absence of radiation exposure. On ultrasound examination in B-mode, AVMs can have a nidus-like appearance or present multiple non-compressible vascular spaces. The color-Doppler and the Power Doppler are particularly useful for a better definition of the flow characteristics in the afferent arteries, in the nidus and in the efferent drainage veins. AVMs have multidirectional blood flow (high-flux Doppler signal with low-resistance arterial waveforms), highamplitude arterial waveforms with spectrum widening and "arterialized" venous outflow. Typical is also the finding of "aliasing" inside the nidus which attests the presence of turbulent flow. Ultrasound examination allows to identify the exact location of the AVM and the involved tissue plan as well as to map the origin of the afferent vessels of the normal arterial system and drainage into the normal venous system. Furthermore, the rate of the arterialized flow within the venous system must also be identified. However ultrasound has some limitations regarding the definition of the extent of the lesion, which can 
be overcome by using CT which can provide useful informations on the involvement of adjacent organs (Botta et al., 2020). Furthermore, CT with contrast enhancer is able to give an accurate mapping and measurement of arteries, veins and nidus, as well as guaranteeing an assessment of flow patterns for the use of interventional radiology or for surgical planning (Lee et al., 2015).

\section{Conclusions}

AVM are high-flow vascular malformations that can occur in different locations, although intracerebral localization appears to be the most frequent in children. We have described a rare case of adrenal AVM that was incidentally diagnosed in a child who had no lesion-related symptoms and who was undergoing diagnostic investigation for another reason. Imaging, in particular, ultrasound and color-Doppler, together with CT with contrast enhancer, have had in this case an important role in the diagnosis of the pathology.

Conflict of Interest: The Authors declare that they have no conflict of interest.

Informed Consent: All procedures followed were in accordance with the ethical standards of the responsible committee on human experimentation (institutional and national) and with the Helsinki Declaration of 1975, and its late amendments. Additional informed consented was obtained from all patients for which identifying information is not included in this article.

Human and Animal Rights: This article does not contain any studies with human or animal subjects performed by any of the Authors.

Patient Consent: The patient's name has been removed from all images and cannot be recognized in any way.

\section{References}

Botta F, Raimondi S, Rinaldi L, Bellerba F, Corso F, Bagnardi V, Origgi D, Minelli R, Pitoni G, Petrella F, Spaggiari L. Association of a CT-Based Clinical and Radiomics Score of Non-Small Cell Lung Cancer (NSCLC) with Lymph Node Status and Overall Survival. Cancers 2020; 12: 1432.

Brillantino C, Rossi E, Minelli R, Irace D, Castelli L, Zeccolini R, Bignardi E, Tufano A. A rare case of renal tumor in children: clear cell sarcoma. G Chir 2019; 40: 217-224.

Chen W, Choi EJ, McDougall CM, Su H. Brain arteriovenous malformation modeling, pathogenesis, and novel therapeutic targets. Transl Stroke Res 2014;5: 316-329.

Conway AM and Rosen RJ. Arteriovenous Malformations: Syndrome Identification and Vascular Management. Curr Treat Options Cardiovasc Med 2018; 20: 67.

Dubois J and Garel L. Imaging and therapeutic approach of hemangiomas and vascular malformations in the pediatric 
age group. Pediatr Radiol 1999; 29: 879-893.

Kollipara R, Odhav A, Rentas KE, Rivard DC, Lowe LH, Dinneen L. Vascular Anomalies in Pediatric Patients Updated Classification, Imaging, and Therapy. Radiol Clin N Am 2013; 51: 659-672.

Lee BB, Antignani PL, Baraldini V, Baumgartner I, Berlien P, Blei F, Carrafiello GP, Grantzow R, Ianniello A, Laredo J, Loose D, Lopez Gutierrez JC, Markovic J, Mattassi R, Parsi K, Rabe E, Roztocil K, Shortell C, Vaghi M, ISVI-IUA consensus document diagnostic guidelines of vascular anomalies: vascular malformations and hemangiomas. Int Angiol 2015; 34: 333374.

Ozpinar A, Mendez G, Abla AA. Epidemiology, genetics, pathophysiology, and prognostic classifications of cerebral arteriovenous malformations. Handb Clin Neurol 2017; 143: 5-13.

Rangel-Castilla L, Russin JJ, Martinez-Del-Campo E, Soriano-Baron H, Spetzler RF, Nakaji P. Molecular and cellular biology of cerebral arteriovenous malformations: a review of current concepts and future trends in treatment. Neurosurg Focus 2014; 37: E1.

Samadi K and Salazar GM, Role of imaging in the diagnosis of vascular malformations vascular malformations. Cardiovasc Diagn Ther 2019; 9: S143-S151.

Stapf C, Mohr JP, Pile-Spellman J, Solomon RA, Sacco RL, Connolly ES. Epidemiology and natural history of arteriovenous malformations. Neurosurg Focus 2001; 11: e1.

Tufano A, Minelli R, Rossi E, Brillantino C, Di Serafino M, Zeccolini M, Cantisani V, Vallone G. Inferior epigastric artery pseudoaneurysm secondary to port placement during a robot-assisted laparoscopic radical cystectomy. J Ultrasound 2020; 11 : $1-4$. 